\title{
Reaction of laying hens to low phosphorus diets and addition of different phytase preparations
}

\author{
D. Jamroz ${ }^{1}$, J. Orda ${ }^{1}$, J. Skorupińska ${ }^{1}$, A.Wiliczkiewicz ${ }^{1}$, \\ T. Wertelecki ${ }^{1}$, R. Żyłka ${ }^{2}$ and A.-M. Klünter ${ }^{3}$
}

\author{
Wroctaw Agricultural University \\ ${ }^{1}$ Department of Animal Nutrition and Feed Quality \\ Chetmońskiego 38D, 51-630 Wroctaw, Poland \\ ${ }^{2}$ Department of Physics and Biophysics \\ Norwida 25/27, 50-375 Wrockaw, Poland \\ ${ }^{3}$ CRNA, Societe Chimique Roche SA \\ Saint Louis Cedex, France
}

(Received 18 April 2002; revised version 20 November 2002; accepted 20 December 2002)

\begin{abstract}
The purpose of the presented study was to determine the reaction of hens to low-phosphorus diets and supplementation with different microbial phytases by assessing laying performance, feed intake, egg shell quality, as well as bone strength parameters and bone contents of $\mathrm{Ca}, \mathrm{P}, \mathrm{Mg}$, and $\mathrm{Zn}$. At the age of 16 weeks, 288 Lohmann Brown pullets were allocated to 6 dietary treatments, each treatment group consisting of 12 cages, 4 birds per cage. Before the laying period the pullets were fed with a standard diet containing $150 \mathrm{~g}$ crude protein (CP) $11.3 \mathrm{MJ} \mathrm{ME}, 4 \mathrm{~g}$ available $\mathrm{P}$ and $10.6 \mathrm{~g}$ of $\mathrm{Ca}$ per $\mathrm{kg}$ feed. The experimental diets fed from the first day of laying were based on wheat and barley and contained $165 \mathrm{~g} \mathrm{CP}$ and $11.2 \mathrm{MJ} \mathrm{ME} / \mathrm{kg}$. In diets I to V the level of available P was lowered to $1.89 \mathrm{~g} / \mathrm{kg}$ in the first period of laying and to $1.31 \mathrm{~g} / \mathrm{kg}$ in second and third periods, while in the control (VI) diet the amount of available P was $3.09 \mathrm{~g} / \mathrm{kg}$. In diets II-V two kinds of phytase were applied at levels of 300 or $450 \mathrm{U} / \mathrm{kg}$ of diet. The phosphorus level did not affect the laying rate. The higher P level enhanced egg shell thickness. Supplementation with phytase did not affect the performance of hens. Improved feed conversion was noted only when the diet was supplemented with $450 \mathrm{U}$ of phytase B per kg. Phytase supplementation of low-P diets significantly improved the strength and elasticity of the tibia and femur, which in groups II-V were on a similar level as in the positive control group.
\end{abstract}

KEY WORDS: microbial phytase, performance, egg shell quality, bone quality, laying hens 


\section{INTRODUCTION}

Plant compounds that are used in formulating mixtures for poultry contain sufficient amounts of phosphorus to cover the birds' requirements. However, about $60-70 \%$ of total $\mathrm{P}$ exists in the form of phytic acid, which makes the phosphorus unavailable or hardly available for poultry (Oloffs et al., 2000; Liebert et al., 2001).

The presence of phytic acid in feed components worsens the absorption of both trace- and macroelements from the intestinal digesta (Van der Klis, 1991), which may lead to their deficit (De Groote and Huyghebaert, 1997). Phytic acid may bind some proteins and form stable phytate-protein complexes that can lower the utilization of proteins and amino acids. Furthermore, such complexes with intestinal proteases may inhibit protein degradation and digestion (Yi et al., 1996b; Szkudelski, 1997; Ravindran et al., 1999).

Microrganisms are able to hydrolyse phytates; phytase-myo-inositol hexaphosphate-phospho-hydrolase originating from microbes is an enzyme that releases phosphorus from phytate complexes (Żyła et al., 1989, 1996; Simons et al., 1990; Kornegay et al., 1996; Hadorn and Wiedmer, 1998). The activity of phytase produced by intestinal microorganisms is low, also the native phytases of wheat, barley or their by-products are not sufficient for significant improvement of phosphorus utilization (Oloffs et al., 2000; Liebert et al., 2001). Supplementation of diets with microbial phytase preparations can improve the bioavailability of phosphorus from dietary phytate (Sebastian et al., 1996; Leske and Coon, 1999; Saynaeve et al., 2000), increase $\mathrm{P}$ utilization and digestion, protein utilization, and enhance $\mathrm{N}$ retention in broilers and laying hens (Yi et al., 1996a; Szkudelski, 1997). Beneficial effects of phytases on phosphorus utilization, bone strength, and egg shell quality were also found in other experiments on hens (Um and Paik, 1999; Gordon and Short, 2001).

The purpose of the present study was to determine the response of laying hens to low-phosphorus diets supplemented with two levels of different phytase preparations. Laying performance, main parameters of egg shell quality, bone composition and strength parameters were investigated.

\section{MATERIAL AND METHODS}

The experiment was performed with 288 Lohmann Brown layers. At the age of 16 weeks pullets were transferred to cages, 4 pullets per cage and allocated to 6 treatments, each encompassing 12 cages. The average initial body weight of birds was similar in all groups. Feed and water were supplied ad libitum. The temperature in the laying house varied from 18 to $26^{\circ} \mathrm{C}$ during the warm summer days. The lighting program was $10 \mathrm{~h}$ darkness and $14 \mathrm{~h}$ light with an intensity of about $30 \mathrm{~lx}$. 
Before the laying period, pullets were fed a standard diet containing $150 \mathrm{~g}$ crude protein and $11.3 \mathrm{MJ} \mathrm{ME} / \mathrm{kg}$

Three diets were prepared to contain 4.01 or 3.29 total P per kg (low-phosphorus diet LP-1 and LP-2) and $5.5 \mathrm{~g}$ P per kg (control diet) (Table 1).

TABLE 1

Composition of diets, $\mathrm{g} / \mathrm{kg}$

\begin{tabular}{lccc}
\hline & & Diets & \\
\cline { 2 - 4 } Components & LP-1 & LP-2 & control \\
\hline Barley var. Rudzik & 300 & 300 & 300 \\
Winter wheat var. Cobra & 414 & 414 & 366 \\
Soya oil & 16 & 16 & 30 \\
Soyabean oilmeal & 178 & 178 & 189 \\
Dicalcium phosphate & 4 & - & 13 \\
Limestone & 74 & 78 & 88 \\
Mineral-vitamin premix ${ }^{1}$ & 14 & 14 & 14 \\
Calculated & & & \\
metabolizable energy, MJ/kg & 11.20 & 11.20 & 11.20 \\
crude protein (N x 6.25) & 165.0 & 165.0 & 165.2 \\
methionine & 3.53 & 3.53 & 3.52 \\
lysine & 8.95 & 8.95 & 9.18 \\
Ca & 30.00 & 31.47 & 37.00 \\
P & 4.01 & 3.29 & 5.50 \\
P available & 1.89 & 1.31 & 3.09 \\
Mg & 1.32 & 1.32 & 1.32 \\
Zn, mg/kg & 83.1 & 84.1 & 80.3 \\
\hline
\end{tabular}

${ }^{1}$ supplied per kg diet: retinol acetate $10.000 \mathrm{IU}$; cholekalciferol $2.000 \mathrm{IU}$; mg: tocopherol 15; menadion 1.5; thiamine 2; riboflavin 4; pyridoxine 1.0 ; cyanocobalamin 0.01 ; nicotinic acid 30 ; folic acid 0.5 ; panthothenic acid 4; choline chloride 250; Mn 60; Zn 50; Fe 30; Cu 5; I 0.5; Se 0.12; methionine $1 \mathrm{~g}$ and $\mathrm{NaCl} 4 \mathrm{~g}$

${ }^{2}$ calculated on the basis of P-availability from Nutrient Requirements (1996)

Diets LP-1 and LP-2 were fed either unsupplemented or supplemented with two phytase preparations at a level of 300 or 450 phytase units per $\mathrm{kg}$ (Table 2). All diets were fed ad libitum in mash form. Group VI was fed the control diet from week 19 of life to the end of the experiment (298 days). From week 1 to 14 week of the laying period groups I to V were fed diet LP-1 that was either unsupplemented or supplemented, thereafter until the end of the experiment respective diets LP-2 containing $1.31 \mathrm{~g}$ of available P per kg.

Laying performance was determined during 298 days of laying. The number of eggs was recorded daily, all eggs from each cage were weighed once a week. Feed intake, cleanness and abnormalities of the egg shell and mortality of birds were recorded during the laying period. 
TABLE 2

Design of experiment

\begin{tabular}{cccc}
\hline Group & & Diet & Phytase $^{2}, \mathrm{U} / \mathrm{kg}$ diet \\
\hline & I & LP-1 and LP-2 & No supplement \\
& II & LP-1 and LP-2 & $+\mathrm{A}^{1} 300$ \\
III & LP-1 and LP-2 & + A 450 \\
IV & LP-1 and LP-2 & $+\mathrm{B}^{1} 300$ \\
V & LP-1 and LP-2 & + B 450 \\
VI & Control diet & No supplement \\
\hline
\end{tabular}

${ }^{1}$ A (NATUPHOS ${ }^{\circledR} 5000 \mathrm{G}$, lot NHPG 1596740 phytase U/g B (RONOZYME® P (CT), lot HB 9000033133 phytase U/g

${ }^{2}$ one unit of phytase activity is defined as the amount that releases $1 \mu \mathrm{mol}$ of inorganic phosphate from $5.0 \mathrm{mmol}$ sodium phytate per minute at $\mathrm{pH} 5.5$ in $37^{\circ} \mathrm{C}$

During weeks 13, 19, and 33 from the start of the experiment (measurement periods 1,2, and 3) the quality of eggs and bones and the content of $\mathrm{Ca}, \mathrm{P}, \mathrm{Mg}$ and $\mathrm{Zn}$ in bones were estimated.

Thirty eggs were randomly selected from each group to determine the weight of whole eggs. Egg analyses were performed using standard procedures with a PM600 PX-processor apparatus (Technical Service and Supplies Ltd., York, England). Shell thickness was measured in three points using a Modul with Micrometric screw (Mitutoys). Each measurement was carried out in duplicate. Shell strength was determined under a maximum pressure of $3.5 \mathrm{~kg}$.

In each measurement period one hen from each cage was randomly chosen, weighed and killed. From five selected animals per group the tibia and femur bones from the left leg were prepared for evaluation of strength and elasticity. Analysis of physical properties was done on fresh bones that had been stored in a refrigerator at a temperature of $4-5^{\circ} \mathrm{C}$. Bone deflection (h) was measured by a standard method, in which the force (Fn) was applied to the shaft of the bone supported at both epiphyses, taking into account Hook's law concerning bone elasticity, where the vector of deflection is proportional to the supported force $(\mathrm{Fn}=\alpha \mathrm{xh}$ ), and $\alpha$ is the coefficient of elasticity.

At the breaking point, the inner and the outer diameter as well as the cortex thickness of the bone were measured using a slide calliper assuming that the bone cross section was circular. Measurements were taken from ten different, randomly chosen points. On the basis of the obtained data, the surface of bone and the cross section were determined. The elasticity of the bones was estimated by a mediation model:

$$
\alpha=E \frac{\pi\left(D^{4}-d^{4}\right)}{6 L^{3}}
$$


where:

D - external diameter

d - internal diameter

L - distance between support points

E - Young's Modulus.

The parameters were evaluated according to variable force (from low to high pressure) up to the bone breaking point. Maximal loading was compared with the strength coefficient of long bones (W). Finally, the coefficient of elasticity was estimated by comparing two factors: deflection and breaking force: $\alpha=\Delta \mathrm{F} \mathrm{n} / \Delta \mathrm{h}$. All estimated parameters of bone quality were expressed in relation to $100 \mathrm{~g}$ of metabolic body weight.

The chemical composition of the diets was determined according to standard methods (AOAC, 1990). The $\mathrm{Ca}, \mathrm{P}, \mathrm{Mg}$ and $\mathrm{Zn}$ contents were determined in dried bones prepared from the right legs. After previous mineralization of diets and bones, phosphorus was analysed by the vanadomolybdate method, using a Specol 11 (Carl Zeiss, Jena) spectrophotometer at a wave length of $470 \mathrm{~nm} . \mathrm{Ca}, \mathrm{Mg}$ and $\mathrm{Zn}$ were determined by atomic absorption spectrophotometry using an AAS-3 EA 30 (Carl Zeiss, Jena) apparatus.

The energy value of the diets (Table 1) was calculated according to the European Table (1989).

Statistical calculations were conducted using the Statgraphics Plus ver. 7.0 software. Data was compared using one-way and two-way ANOVA (Brandt, 1997). A split-plot ANOVA was used to test for period $x$ treatment interactions. The significance of differences between groups was examined after data transformation (arcsin) for percentages and $\log _{10}$ for other variables. Tukey's Multiple Range Test was used to determine the significance of differences between treatment means $(\mathrm{P} \leq 0.05$ and $\mathrm{P} \leq 0.01)$.

\section{RESULTS}

In the first measurement period, the body weight of hens averaged $1855 \mathrm{~g}$, except in group I (2052 g); in the second period it averaged $1984 \mathrm{~g}$, in the third, $2072 \mathrm{~g}$. The low phosphorus level in the diets of groups I to $\mathrm{V}$ did not result in significant changes of body weight during the 298 days of the experiment (Table 3). The health status of the birds was very good.

Phosphorus level and phytase addition did not significantly affect laying rate, egg weight, number of laid eggs, or total egg mass, although slightly better results (by $3 \%$ ) in total egg mass were noted in group $\mathrm{V}$, in which the diet was supplemented with phytase B at a rate of $450 \mathrm{U} / \mathrm{kg}$ feed and in group III (phytase A at $450 \mathrm{U} / \mathrm{kg}$ ) 
TABLE 3

Body weight of hens, $\mathrm{g}^{1}$

\begin{tabular}{|c|c|c|c|c|c|c|c|}
\hline \multirow{2}{*}{ Item } & \multicolumn{6}{|c|}{ Group } & \multirow{2}{*}{ SEM } \\
\hline & I & II & III & IV & V & VI & \\
\hline Measurement 1 & 2052 & 1827 & 1855 & 1851 & 1902 & 1840 & 34.2 \\
\hline Measurement 2 & $1877^{\mathrm{a}}$ & $1907^{\mathrm{a}}$ & $2216^{\mathrm{b}}$ & $1893^{\mathrm{a}}$ & $1994^{\mathrm{ab}}$ & $2017^{\mathrm{ab}}$ & 39.4 \\
\hline Measurement 3 & 2128 & 2078 & 2000 & 2154 & 2028 & 2042 & 43.6 \\
\hline \multirow[t]{2}{*}{ Mean for measurement } & \multicolumn{2}{|c|}{1} & \multicolumn{2}{|c|}{2} & \multicolumn{2}{|c|}{3} & \\
\hline & \multicolumn{2}{|c|}{$1888^{\mathrm{a}}$} & \multicolumn{2}{|c|}{$1973^{a b}$} & \multicolumn{2}{|c|}{$2065^{\mathrm{b}}$} & 23.3 \\
\hline
\end{tabular}

TABLE 4

Laying performanc (mean for 298 days)

\begin{tabular}{lccccccc}
\hline \multirow{2}{*}{ Parameters } & \multicolumn{7}{c}{ Group } \\
\cline { 2 - 7 } & I & II & III & IV & V & VI & \\
\hline Laying rate, \% & 92.8 & 92.6 & 93.1 & 92.8 & 93.2 & 91.9 & 0.36 \\
Egg weight, g & 61.0 & 59.6 & 60.9 & 60.7 & 62.2 & 61.2 & 0.36 \\
Total egg mass, kg/hen & 16.9 & 16.4 & 16.9 & 16.7 & 17.3 & 16.9 & 0.12 \\
Feed intake, g/bird /day & $119.0^{\mathrm{A}}$ & $124.3^{\mathrm{AB}}$ & $129.4^{\mathrm{B}}$ & $117.0^{\mathrm{A}}$ & $119.9^{\mathrm{A}}$ & $122.4^{\mathrm{AB}}$ & 0.74 \\
Feed intake, kg/kg egg mass & $2.11^{\mathrm{A}}$ & $2.26^{\mathrm{A}}$ & $2.29^{\mathrm{A}}$ & $2.11^{\mathrm{A}}$ & $2.07^{\mathrm{B}}$ & $2.15^{\mathrm{A}}$ & 0.02 \\
Cracked eggs, \% & 2.16 & 1.97 & 2.52 & 2.58 & 2.77 & 1.65 & 0.24 \\
Deformed eggs, \% & $0.01^{\mathrm{a}}$ & $0.01^{\mathrm{a}}$ & $0.14^{\mathrm{ab}}$ & $0.01^{\mathrm{a}}$ & $0.01^{\mathrm{a}}$ & $0.26^{\mathrm{b}}$ & 0.04 \\
\hline
\end{tabular}

${ }_{\mathrm{a}, \mathrm{b}, \mathrm{A}, \mathrm{B}}$ means in a row with no common superscripts are significantly different: ${ }^{\mathrm{a}, \mathrm{b}} \mathrm{P}<0.05$; $^{\mathrm{A}, \mathrm{B}} \mathrm{P}<0.01$

(Table 4). Feed conversion calculated per kg of egg mass was significantly lower in group V than in other groups. Differences in the percentage of cracked or deformed eggs among groups were relatively low and statistically not significant (Table 4).

Egg shell thickness in group VI was higher than in group I; in the first and second periods these differences were highly significant $(\mathrm{P} \leq 0.01)$, however in the third period (in older hens) no significant difference was found. In young hens (in the first period) egg shell thickness was significantly improved by addition of phytase A at $300 \mathrm{U} / \mathrm{kg}$ to the diet and in the second period, by phytase B at 450 $\mathrm{U} / \mathrm{kg}$ (Table 5).

The strength and elasticity of the tibia and femur are presented in Tables 6 and 7. Similarly as egg shell quality, large individual variation of the analysed bone parameters were observed. The most important parameters of bone quality, i.e. strength $(\mathrm{N})$ and strength to surface ratio, indicate that the phosphorus content in 
TABLE 5

Parameters of egg shell quality in three measurement periods

\begin{tabular}{|c|c|c|c|c|c|c|c|}
\hline \multirow{2}{*}{ Parameters } & \multicolumn{6}{|c|}{ Group } & \multirow{2}{*}{ SEM } \\
\hline & I & II & III & IV & V & VI & \\
\hline \multicolumn{8}{|c|}{ Measurement 1} \\
\hline Laying rate, $\%$ & 95.0 & 97.0 & 96.3 & 95.5 & 94.0 & 95.7 & \\
\hline Egg weight, g & $61.7^{\mathrm{ab}}$ & $59.6^{\mathrm{Aa}}$ & $61.8^{\mathrm{ab}}$ & $61.3^{\mathrm{ab}}$ & $62.3^{\mathrm{b}}$ & $62.5^{\mathrm{B}}$ & 0.31 \\
\hline Egg shell strength, kg & $3.14^{\mathrm{A}}$ & $3.18^{\mathrm{A}}$ & $3.04^{\mathrm{A}}$ & $2.99^{\mathrm{AB}}$ & $2.82^{\mathrm{AB}}$ & $2.56^{\mathrm{B}}$ & 0.05 \\
\hline Egg shell thickness, mm & $0.326^{\mathrm{A}}$ & $0.342^{\mathrm{B}}$ & $0.326^{\mathrm{A}}$ & $0.324^{\mathrm{A}}$ & $0.319^{\mathrm{A}}$ & $0.340^{\mathrm{B}}$ & 0.01 \\
\hline \multicolumn{8}{|c|}{ Measurement 2} \\
\hline Laying rate, $\%$ & 95.0 & 92.5 & 94.6 & 96.7 & 94.2 & 95.2 & \\
\hline Egg weight, $g$ & $64.1^{\mathrm{ab}}$ & $62.5^{\mathrm{a}}$ & $64.6^{\mathrm{ab}}$ & $63.5^{\mathrm{ab}}$ & $65.0^{\mathrm{b}}$ & $65.1^{\mathrm{b}}$ & 0.32 \\
\hline Egg shell strength, kg & $3.12^{\mathrm{A}}$ & $3.05^{\mathrm{a}}$ & $2.88^{\mathrm{ab}}$ & $2.79^{\mathrm{ab}}$ & $2.62^{\mathrm{Bb}}$ & $3.08^{\mathrm{A}}$ & 0.05 \\
\hline Egg shell thickness, mm & $0.289^{\mathrm{A}}$ & $0.286^{\mathrm{A}}$ & $0.298^{\mathrm{A}}$ & $0.303^{\mathrm{A}}$ & $0.367^{\mathrm{B}}$ & $0.359^{\mathrm{B}}$ & 0.01 \\
\hline \multicolumn{8}{|c|}{ Measurement 3} \\
\hline Laying rate, $\%$ & 90.6 & 86.3 & 86.5 & 88.6 & 93.1 & 92.0 & \\
\hline Egg weight, g & 64.9 & 64.4 & 64.4 & 64.9 & 65.3 & 65.4 & 0.34 \\
\hline Egg shell strength, kg & 2.57 & 2.61 & 2.46 & 2.60 & 2.31 & 2.53 & 0.05 \\
\hline Egg shell thickness, mm & $0.358^{\mathrm{a}}$ & $0.357^{\mathrm{a}}$ & $0.377^{\mathrm{b}}$ & $0.355^{\mathrm{a}}$ & $0.357^{\mathrm{a}}$ & $0.362^{\mathrm{a}}$ & 0.02 \\
\hline \multicolumn{8}{|c|}{ Means for three measurements } \\
\hline Egg weight, g & $63.6^{\mathrm{ab}}$ & $62.2^{\mathrm{Aa}}$ & $63.6^{\mathrm{ab}}$ & $63.2^{\mathrm{ab}}$ & $64.2^{\mathrm{Bb}}$ & $64.3^{\mathrm{B}}$ & 0.31 \\
\hline Egg shell strenght, kg & $2.94^{\mathrm{A}}$ & $2.95^{\mathrm{A}}$ & $2.79^{\mathrm{AB}}$ & $2.79^{\mathrm{AB}}$ & $2.58^{\mathrm{B}}$ & $2.72^{\mathrm{AB}}$ & 0.05 \\
\hline Egg shell thickness, mm & $0.324^{\mathrm{A}}$ & $0.328^{\mathrm{A}}$ & $0.337^{\mathrm{ABC}}$ & $0.327^{\mathrm{AB}}$ & $0.347^{\mathrm{BC}}$ & $0.354^{\mathrm{C}}$ & 0.01 \\
\hline Means for measurements & \multicolumn{2}{|l|}{1} & \multicolumn{2}{|l|}{2} & \multicolumn{2}{|l|}{3} & \\
\hline egg weight, g & \multicolumn{2}{|c|}{$61.5^{\mathrm{A}}$} & \multicolumn{2}{|c|}{$64.1^{\mathrm{B}}$} & \multicolumn{2}{|c|}{$64.9^{\mathrm{B}}$} & 0.20 \\
\hline egg shell strength, $\mathrm{kg}$ & \multicolumn{2}{|c|}{$2.95^{\mathrm{A}}$} & \multicolumn{2}{|c|}{$2.92^{\mathrm{B}}$} & \multicolumn{2}{|c|}{$2.51^{\mathrm{C}}$} & 0.03 \\
\hline egg shell thickness, $\mathrm{mm}$ & \multicolumn{2}{|c|}{$0.33^{\mathrm{A}}$} & \multicolumn{2}{|c|}{$0.32^{\mathrm{B}}$} & \multicolumn{2}{|c|}{$0.37^{\mathrm{C}}$} & 0.00 \\
\hline
\end{tabular}

the diet and supplementation with phytase significantly affected these parameters in the first and third periods (strength to surface ratio). A clear tendency towards improvement of bone strength was observed particularly in the positive control group (VI) in comparison with groups I-V.

The parameters of bone strength $\left(\mathrm{N} / \mathrm{mm}^{2}\right)$ and elasticity were improved in hens fed diets supplemented with phytases in comparison with the low-P group (I). Addition of phytases to the low phosphorus diet improved both tibia and femur strength and elasticity in nearly all cases (except group II). The effects were slightly better with higher phytase dosages. The best tibia quality was found in hens from group V and femur quality in groups III and V.

The average values of the analysed parameters for the tibia and femur in subsequent measurement periods indicate that in older birds these parameters were higher in both the low P (I) and the positive control group (VI). 
TABLE 6

Parameters of tibia bones of hens calculated per $100 \mathrm{~g}$ of metabolic body weight $\left(\mathrm{BW}^{0.67}\right)$

\begin{tabular}{|c|c|c|c|c|c|c|c|}
\hline \multirow{2}{*}{ Parameters } & \multicolumn{6}{|c|}{ Group } & \multirow{2}{*}{ SEM } \\
\hline & I & II & III & IV & $\mathrm{V}$ & VI & \\
\hline \multicolumn{8}{|c|}{ Measurement 1} \\
\hline Cortex thickness, mm & 0.49 & 0.52 & 0.47 & 0.46 & 0.57 & 0.56 & 0.02 \\
\hline Surface, $\mathrm{mm}^{2}$ & 10.23 & 11.70 & 10.17 & 9.27 & 13.23 & 12.86 & 0.59 \\
\hline Strength, $\mathrm{N}$ & $69.16^{\mathrm{a}}$ & $78.67^{\mathrm{ab}}$ & $74.10^{\mathrm{a}}$ & $74.91^{\mathrm{a}}$ & $79.59^{\mathrm{ab}}$ & $98.99^{b}$ & 3.32 \\
\hline $\begin{array}{l}\text { Strength to surface ratio, } \\
\mathrm{N} / \mathrm{mm}^{2}\end{array}$ & $4.17^{\mathrm{abc}}$ & $4.44^{\mathrm{abc}}$ & $4.82^{\mathrm{abc}}$ & $5.46^{\mathrm{b}}$ & $3.97^{\mathrm{a}}$ & $5.25^{\mathrm{b}}$ & 0.18 \\
\hline $\begin{array}{l}\text { Elasticity alpha - } \\
\text { coefficient, } \mathrm{N} / \mathrm{mm}\end{array}$ & $95.35^{\mathrm{a}}$ & $120.07^{\mathrm{ab}}$ & $113.60^{\mathrm{ab}}$ & $111.25^{\mathrm{ab}}$ & $121.84^{\mathrm{ab}}$ & $131.61^{\mathrm{b}}$ & 5.40 \\
\hline \multicolumn{8}{|c|}{ Measurement 2} \\
\hline Cortex thickness, mm & $0.49^{\mathrm{bc}}$ & $0.40^{\mathrm{a}}$ & $0.43^{\mathrm{ab}}$ & $0.43^{a b}$ & $0.48^{\mathrm{bc}}$ & $0.51^{\mathrm{c}}$ & 0.01 \\
\hline Surface, $\mathrm{mm}^{2}$ & $9.71^{\mathrm{ab}}$ & $8.20^{\mathrm{a}}$ & $8.45^{\mathrm{a}}$ & $9.03^{\mathrm{ab}}$ & $9.92^{\mathrm{ab}}$ & $10.82^{\mathrm{b}}$ & 0.31 \\
\hline Strength, N & $62.49^{\mathrm{a}}$ & $60.15^{\mathrm{a}}$ & $68.24^{\mathrm{a}}$ & $64.27^{\mathrm{a}}$ & $74.60^{\mathrm{b}}$ & $73.78^{\mathrm{b}}$ & 2.38 \\
\hline $\begin{array}{l}\text { Strength to surface ratio, } \\
\mathrm{N} / \mathrm{mm}^{2}\end{array}$ & $4.14^{\mathrm{a}}$ & $4.75^{\mathrm{b}}$ & $4.71^{\mathrm{b}}$ & $4.46^{\mathrm{ab}}$ & $4.65^{\mathrm{b}}$ & $4.25^{\mathrm{a}}$ & 0.14 \\
\hline $\begin{array}{l}\text { Elasticity alpha - } \\
\text { coefficient, N/mm }\end{array}$ & 103.59 & 94.76 & 111.83 & 101.53 & 118.60 & 116.84 & 3.90 \\
\hline \multicolumn{8}{|c|}{ Measurement 3} \\
\hline Cortex thickness, mm & 0.51 & 0.43 & 0.49 & 0.43 & 0.47 & 0.45 & 0.01 \\
\hline Surface, $\mathrm{mm}^{2}$ & 10.02 & 9.28 & 9.75 & 9.07 & 9.85 & 9.01 & 0.21 \\
\hline Strength, $\mathrm{N}$ & $68.42^{\mathrm{a}}$ & $61.55^{\mathrm{a}}$ & $79.36^{\mathrm{b}}$ & $82.40^{\mathrm{b}}$ & $70.54^{\mathrm{a}}$ & $81.01^{\mathrm{b}}$ & 2.96 \\
\hline $\begin{array}{l}\text { Strength to surface ratio, } \\
\mathrm{N} / \mathrm{mm}^{2}\end{array}$ & $4.04^{\mathrm{a}}$ & $3.99^{\mathrm{a}}$ & $5.06^{\mathrm{b}}$ & $4.52^{\mathrm{ab}}$ & $4.41^{\mathrm{a}}$ & $5.59 \mathrm{~b}$ & 0.17 \\
\hline $\begin{array}{l}\text { Elasticity alpha - } \\
\text { coefficient, N/mm }\end{array}$ & $116.54^{\mathrm{a}}$ & $113.31^{\mathrm{a}}$ & $169.81^{\mathrm{b}}$ & $118.90^{\mathrm{ab}}$ & $119.78^{\mathrm{ab}}$ & $140.97^{\mathrm{ab}}$ & 6.60 \\
\hline \multicolumn{8}{|c|}{ Means for three measurements } \\
\hline Cortex thickness, mm & 0.49 & 0.45 & 0.46 & 0.44 & 0.51 & 0.51 & 0.01 \\
\hline Surface, $\mathrm{mm}^{2}$ & 9.99 & 9.73 & 9.51 & 9.14 & 11.00 & 10.90 & 0.25 \\
\hline Strength, $\mathrm{N}$ & $66.69^{\mathrm{a}}$ & $66.79^{\mathrm{a}}$ & $73.91^{\mathrm{ab}}$ & $73.94^{\mathrm{ab}}$ & $74.91^{\mathrm{ab}}$ & $84.59^{\mathrm{b}}$ & 1.76 \\
\hline $\begin{array}{l}\text { Strength to surface ratio, } \\
\mathrm{N} / \mathrm{mm}^{2}\end{array}$ & $4.12^{\mathrm{a}}$ & $4.39^{\mathrm{ab}}$ & $4.86^{\mathrm{ab}}$ & $4.87^{\mathrm{ab}}$ & $4.34^{\mathrm{ab}}$ & $5.03^{\mathrm{b}}$ & 0.09 \\
\hline $\begin{array}{l}\text { Elasticity alpha - } \\
\text { coefficient, N/mm }\end{array}$ & 105.07 & 109.48 & 130.37 & 110.66 & 120.07 & 130.67 & 3.30 \\
\hline Means for measurements & \multicolumn{3}{|c|}{1} & \multicolumn{2}{|l|}{2} & \multicolumn{2}{|r|}{3} \\
\hline Cortex thickness, mm & \multicolumn{2}{|c|}{0.51} & \multicolumn{3}{|c|}{0.46} & \multicolumn{2}{|r|}{0.46} \\
\hline Surface, $\mathrm{mm}^{2}$ & \multicolumn{2}{|c|}{$11.24^{\mathrm{A}}$} & \multicolumn{3}{|c|}{$9.40^{\mathrm{B}}$} & \multicolumn{2}{|r|}{$9.50^{\mathrm{B}}$} \\
\hline Strength, $\mathrm{N}$ & \multicolumn{2}{|c|}{$79.24^{\mathrm{A}}$} & \multicolumn{3}{|c|}{$67.33^{\mathrm{B}}$} & \multicolumn{2}{|c|}{$73.38^{\mathrm{AB}}$} \\
\hline $\begin{array}{l}\text { Strength to surface ratio, } \\
\mathrm{N} / \mathrm{mm}^{2}\end{array}$ & \multicolumn{2}{|r|}{4.68} & \multicolumn{3}{|c|}{4.49} & \multicolumn{2}{|c|}{4.59} \\
\hline $\begin{array}{l}\text { Elasticity alpha - } \\
\text { coefficient, N/mm }\end{array}$ & \multicolumn{2}{|c|}{$115.95^{\mathrm{AB}}$} & \multicolumn{3}{|c|}{$108.01^{\mathrm{A}}$} & \multicolumn{2}{|c|}{$128.90^{\mathrm{B}}$} \\
\hline
\end{tabular}


TABLE 7

Parameters of femur bones of hens calculated per $100 \mathrm{~g}$ of metabolic body weight $\left(\mathrm{BW}^{0.67}\right)$

\begin{tabular}{|c|c|c|c|c|c|c|c|}
\hline \multirow{2}{*}{ Parameters } & \multicolumn{6}{|c|}{ Group } & \multirow{2}{*}{ SEM } \\
\hline & I & II & III & IV & $\mathrm{V}$ & VI & \\
\hline \multicolumn{8}{|c|}{ Measurement 1} \\
\hline Cortex thickness, mm & 0.52 & 0.50 & 0.49 & 0.44 & 0.51 & 0.52 & 0.01 \\
\hline Surface, $\mathrm{mm}^{2}$ & 10.77 & 11.71 & 10.67 & 10.16 & 12.49 & 11.46 & 0.38 \\
\hline Strength, N & $74.63^{\mathrm{a}}$ & $78.73^{\mathrm{ab}}$ & $80.43^{\mathrm{ab}}$ & $82.52^{\mathrm{ab}}$ & $86.89^{\mathrm{ab}}$ & $97.44^{\mathrm{b}}$ & 2.81 \\
\hline $\begin{array}{l}\text { Strength to surface ratio, } \\
\mathrm{N} / \mathrm{mm}^{2}\end{array}$ & $4.24^{\mathrm{a}}$ & $4.49^{\mathrm{ab}}$ & $4.98^{\mathrm{ab}}$ & $5.37^{\mathrm{b}}$ & $4.46^{\mathrm{ab}}$ & $5.64^{\mathrm{b}}$ & 0.18 \\
\hline $\begin{array}{l}\text { Elasticity alpha - } \\
\text { coefficient, N/mm }\end{array}$ & $96.82^{\mathrm{a}}$ & $105.95^{\mathrm{ab}}$ & $114.19^{\mathrm{ab}}$ & $99.77^{\mathrm{a}}$ & $102.42^{\mathrm{ab}}$ & $123.31^{\mathrm{b}}$ & 4.20 \\
\hline \multicolumn{8}{|c|}{ Measurement 2} \\
\hline Cortex thickness, mm & $0.50 b^{c}$ & $0.40^{\mathrm{a}}$ & $0.42^{\mathrm{a}}$ & $0.48^{\mathrm{abc}}$ & $0.50^{\mathrm{b}} \mathrm{c}$ & $0.52^{\mathrm{c}}$ & 0.01 \\
\hline Surface, $\mathrm{mm}^{2}$ & $10.65^{\mathrm{a}}$ & $8.92^{\mathrm{b}}$ & $9.52^{\mathrm{ab}}$ & $10.48^{\mathrm{a}}$ & $10.92^{\mathrm{a}}$ & $10.96^{\mathrm{a}}$ & 0.24 \\
\hline Strength, N & $77.60^{\mathrm{a}}$ & $70.95^{\mathrm{ab}}$ & $76.33^{\mathrm{a}}$ & $58.73^{b}$ & $83.49^{\mathrm{a}}$ & $73.37^{\mathrm{ab}}$ & 2.46 \\
\hline $\begin{array}{l}\text { Strength to surface ratio, } \\
\mathrm{N} / \mathrm{mm}^{2}\end{array}$ & $4.65^{\mathrm{ab}}$ & $5.09^{\mathrm{a}}$ & $4.67^{\mathrm{ab}}$ & $3.54^{\mathrm{b}}$ & $4.74^{\mathrm{ab}}$ & $4.13^{\mathrm{ab}}$ & 0.14 \\
\hline $\begin{array}{l}\text { Elasticity alpha - } \\
\text { coefficient, N/mm }\end{array}$ & $84.46^{\mathrm{ab}}$ & $83.88^{\mathrm{ab}}$ & $95.65^{\mathrm{ab}}$ & $75.64^{\mathrm{a}}$ & $103.01^{\mathrm{b}}$ & $91.23^{\mathrm{ab}}$ & 3.00 \\
\hline \multicolumn{8}{|c|}{ Measurement 3} \\
\hline Cortex thickness, mm & 0.47 & 0.47 & 0.47 & 0.44 & 0.54 & 0.46 & 0.02 \\
\hline Surface, $\mathrm{mm}^{2}$ & 10.40 & 10.77 & 10.53 & 10.36 & 11.86 & 10.18 & 0.32 \\
\hline Strength, N & 72.41 & 73.18 & 95.28 & 89.58 & 97.48 & 84.75 & 3.97 \\
\hline $\begin{array}{l}\text { Strength to surface ratio, } \\
\mathrm{N} / \mathrm{mm}^{2}\end{array}$ & $4.10^{\mathrm{a}}$ & $4.06^{\mathrm{a}}$ & $5.62^{\mathrm{b}}$ & $5.06^{\mathrm{ab}}$ & $5.04^{\mathrm{ab}}$ & $5.46^{\mathrm{b}}$ & 0.19 \\
\hline $\begin{array}{l}\text { Elasticity alpha - } \\
\text { coefficient, N/mm }\end{array}$ & $94.18^{\mathrm{a}}$ & $99.47^{\mathrm{ab}}$ & $129.49^{\mathrm{ab}}$ & $134.50^{\mathrm{b}}$ & $130.96^{b}$ & $120.96^{\mathrm{ab}}$ & 6.90 \\
\hline \multicolumn{8}{|c|}{ Means for three measurements } \\
\hline Cortex thickness, mm & 0.50 & 0.46 & 0.46 & 0.45 & 0.51 & 0.50 & 0.01 \\
\hline Surface, $\mathrm{mm}^{2}$ & 10.61 & 10.47 & 10.27 & 10.34 & 11.75 & 10.87 & 0.20 \\
\hline Strength, N & 74.88 & 74.29 & 83.74 & 76.94 & 88.70 & 85.19 & 1.98 \\
\hline $\begin{array}{l}\text { Strength to surface ratio, } \\
\mathrm{N} / \mathrm{mm}^{2}\end{array}$ & 4.33 & 4.55 & 5.08 & 4.66 & 4.73 & 5.08 & 0.10 \\
\hline $\begin{array}{l}\text { Elasticity alpha - } \\
\text { coefficient, N/mm }\end{array}$ & 91.82 & 96.53 & 113.01 & 103.30 & 110.66 & 111.83 & 3.00 \\
\hline Means for measurements & \multicolumn{3}{|c|}{1} & \multicolumn{2}{|l|}{2} & \multicolumn{2}{|c|}{3} \\
\hline Cortex thickness, mm & \multicolumn{2}{|c|}{0.49} & \multicolumn{3}{|c|}{0.47} & \multicolumn{2}{|c|}{0.47} \\
\hline Surface, $\mathrm{mm}^{2}$ & \multicolumn{2}{|c|}{11.21} & \multicolumn{3}{|c|}{10.27} & \multicolumn{2}{|c|}{10.65} \\
\hline Strength, N & \multicolumn{2}{|c|}{$83.44^{\mathrm{a}}$} & \multicolumn{3}{|c|}{$73.31^{\mathrm{b}}$} & \multicolumn{2}{|c|}{$84.67^{\mathrm{a}}$} \\
\hline $\begin{array}{l}\text { Strength to surface ratio, } \\
\mathrm{N} / \mathrm{mm}^{2}\end{array}$ & \multicolumn{2}{|c|}{4.86} & \multicolumn{3}{|c|}{4.46} & \multicolumn{2}{|c|}{4.86} \\
\hline $\begin{array}{l}\text { Elasticity alpha - } \\
\text { coefficient, N/mm }\end{array}$ & \multicolumn{2}{|c|}{$107.13^{\mathrm{a}}$} & \multicolumn{3}{|c|}{$88.88^{\mathrm{Ab}}$} & \multicolumn{2}{|c|}{$117.43^{\text {в }}$} \\
\hline
\end{tabular}

a,b,A,B means in a row with no common superscripts are significantly different: ${ }^{a, b} \mathrm{P}<0.05$; ${ }^{A, B} \mathrm{P}<0.01$ 
Content of crude ash in tiba bone and content of minerals in crude ash, $\%$

\begin{tabular}{|c|c|c|c|c|c|c|c|}
\hline \multirow{2}{*}{ Parameters } & \multicolumn{6}{|c|}{ Group } & \multirow{2}{*}{ SEM } \\
\hline & I & II & III & IV & $\mathrm{V}$ & VI & \\
\hline \multicolumn{8}{|c|}{ Measurement 1} \\
\hline Crude ash, $\%$ & $45.5^{\mathrm{a}}$ & $47.2^{\mathrm{ab}}$ & $48.7^{\mathrm{b}}$ & $47.0^{\mathrm{ab}}$ & $46.8^{\mathrm{ab}}$ & $47.3^{\mathrm{ab}}$ & 0.34 \\
\hline \multicolumn{8}{|l|}{ In crude ash } \\
\hline $\mathrm{Ca}$ & 52.3 & 49.2 & 50.6 & 51.4 & 51.1 & 51.7 & 4.86 \\
\hline $\mathrm{P}$ & 36.5 & 34.9 & 34.7 & 34.9 & 35.5 & 35.8 & 2.84 \\
\hline $\mathrm{Mg}$ & 1.18 & 1.15 & 1.05 & 1.07 & 1.05 & 1.14 & 0.17 \\
\hline $\mathrm{Zn}, \mathrm{ppm}$ & 684.2 & 710.2 & 700.1 & 654.6 & 627.4 & 748.8 & 19.4 \\
\hline \multicolumn{8}{|c|}{ Measurement 2} \\
\hline Crude ash, $\%$ & $51.6^{\mathrm{ab}}$ & $48.4^{\mathrm{a}}$ & $51.3^{\mathrm{ab}}$ & $49.3^{\mathrm{ab}}$ & $52.1^{\mathrm{b}}$ & $50.3^{\mathrm{ab}}$ & 0.39 \\
\hline \multicolumn{8}{|l|}{ In crude ash } \\
\hline $\mathrm{Ca}$ & $52.6^{\mathrm{ab}}$ & $57.0^{\mathrm{a}}$ & $52.7^{\mathrm{ab}}$ & $52.3^{\mathrm{ab}}$ & $50.1^{\mathrm{ab}}$ & $47.2^{\mathrm{b}}$ & 9.25 \\
\hline $\mathrm{P}$ & $31.8^{\mathrm{ab}}$ & $35.0^{\mathrm{a}}$ & $32.8^{\mathrm{ab}}$ & $33.4^{\mathrm{ab}}$ & $31.0^{\mathrm{b}}$ & $33.0^{\mathrm{ab}}$ & 4.06 \\
\hline $\mathrm{Mg}$ & 0.93 & 0.97 & 0.88 & 0.97 & 0.90 & 0.90 & 0.14 \\
\hline $\mathrm{Zn}, \mathrm{ppm}$ & 708.5 & 773.6 & 743.5 & 746.5 & 684.3 & 780.5 & 13.07 \\
\hline \multicolumn{8}{|c|}{ Measurement 3} \\
\hline Crude ash, $\%$ & $50.0^{\mathrm{ab}}$ & $46.7^{\mathrm{ab}}$ & $50.9^{\mathrm{a}}$ & $49.0^{\mathrm{ab}}$ & $46.4^{\mathrm{b}}$ & $49.9^{\mathrm{ab}}$ & 0.59 \\
\hline \multicolumn{8}{|l|}{ In crude ash } \\
\hline $\mathrm{Ca}$ & $48.1^{\mathrm{a}}$ & $53.2^{\mathrm{b}}$ & $48.0^{\mathrm{a}}$ & $49.8^{\mathrm{ab}}$ & $53.7^{\mathrm{b}}$ & $50.7^{\mathrm{ab}}$ & 6.66 \\
\hline $\mathrm{P}$ & $32.2^{\mathrm{a}}$ & $34.4^{\mathrm{ab}}$ & $31.1^{\mathrm{a}}$ & $34.0^{\mathrm{ab}}$ & $38.5^{\mathrm{b}}$ & $34.6^{\mathrm{ab}}$ & 7.13 \\
\hline $\mathrm{Mg}$ & $0.90^{\mathrm{ab}}$ & $1.01^{\mathrm{Ac}}$ & $0.82^{\mathrm{Ba}}$ & $0.94^{b}$ & $0.98^{\mathrm{Abc}}$ & $0.98^{\mathrm{bc}}$ & 0.16 \\
\hline $\mathrm{Zn}, \mathrm{ppm}$ & $658.4^{\mathrm{a}}$ & $768.2^{\mathrm{b}}$ & $717.1^{\mathrm{ab}}$ & $723.9^{a b}$ & $807.4^{\mathrm{b}}$ & $792.1^{\mathrm{b}}$ & 15.7 \\
\hline \multicolumn{8}{|c|}{ Means for three measurements } \\
\hline Crude ash, \% & $49.0^{\mathrm{AB}}$ & $47.4^{\mathrm{A}}$ & $50.3^{\mathrm{B}}$ & $48.4^{\mathrm{AB}}$ & $48.4^{\mathrm{AB}}$ & $49.2^{\mathrm{AB}}$ & 0.10 \\
\hline $\mathrm{Ca}$ & 51.0 & 53.2 & 50.4 & 51.2 & 51.6 & 49.8 & 0.30 \\
\hline $\mathrm{P}$ & 33.5 & 34.8 & 32.9 & 34.1 & 35.0 & 34.4 & 4.13 \\
\hline $\mathrm{Mg}$ & $1.00^{\mathrm{a}}$ & $1.04^{\mathrm{Aa}}$ & $0.92^{\mathrm{Bb}}$ & $0.99^{\mathrm{ab}}$ & $0.98^{\mathrm{ab}}$ & $1.01^{\mathrm{a}}$ & 3.06 \\
\hline $\mathrm{Zn}, \mathrm{ppm}$ & 683.7 & 750.7 & 720.2 & 708.3 & 706.4 & 773.8 & 9.68 \\
\hline Means for measurements & \multicolumn{2}{|c|}{1} & \multicolumn{3}{|c|}{2} & \multicolumn{2}{|l|}{3} \\
\hline Crude ash, $\%$ & \multicolumn{2}{|c|}{$47.1^{\mathrm{Aa}}$} & \multicolumn{3}{|c|}{$50.5^{\mathrm{Bb}}$} & \multicolumn{2}{|c|}{$48.8^{c}$} \\
\hline $\mathrm{Ca}$ & \multicolumn{2}{|c|}{51.0} & \multicolumn{3}{|c|}{52.0} & \multicolumn{2}{|l|}{50.6} \\
\hline $\mathrm{P}$ & \multicolumn{2}{|c|}{$35.4^{\mathrm{A}}$} & \multicolumn{3}{|c|}{$32.8^{\mathrm{B}}$} & \multicolumn{2}{|c|}{$34.1^{\mathrm{AB}}$} \\
\hline $\mathrm{Mg}$ & \multicolumn{2}{|c|}{$1.11^{\mathrm{A}}$} & \multicolumn{3}{|c|}{$0.92^{\mathrm{B}}$} & \multicolumn{2}{|c|}{$0.94^{\mathrm{B}}$} \\
\hline $\mathrm{Zn}, \mathrm{ppm}$ & \multicolumn{2}{|c|}{687.6} & \multicolumn{3}{|c|}{739.5} & \multicolumn{2}{|c|}{744.5} \\
\hline
\end{tabular}

Similarly to tibia quality, supplementation of feed with phytases also positively influenced femur quality in all cases. The reduction of the phosphorus level in diet I decreased the bone quality parameters only in the second and third experimental periods. All calculated interactions were insignificant. 
The content of dry matter in the bones was similar in all groups (average 92\%). The crude ash content ranged from about 44 to $52 \%$ and significant differences between groups were noted (Tables 8 and 9), therefore the concentration of minerals was calculated in relation to crude ash. Tibia and femur ash contained from 48 to $57 \% \mathrm{Ca}$, and from 31 to $36.6 \% \mathrm{P}$. Only in some cases were the differences between groups significant. Relatively high variability was found in the $\mathrm{Mg}$ and $\mathrm{Zn}$ contents.

TABLE 9

Content of crude ash in femur bone and content of minerals in crude ash, $\%$

\begin{tabular}{|c|c|c|c|c|c|c|c|}
\hline \multirow{2}{*}{ Parameters } & \multicolumn{6}{|c|}{ Group } & \multirow{2}{*}{ SEM } \\
\hline & I & II & III & IV & V & VI & \\
\hline \multicolumn{8}{|c|}{ Measurement 1} \\
\hline Crude ash, $\%$ & $44.0^{\mathrm{a}}$ & $45.5^{\mathrm{ab}}$ & $46.7^{\mathrm{ab}}$ & $46.8^{\mathrm{ab}}$ & $47.9^{\mathrm{ab}}$ & $50.3^{\mathrm{b}}$ & 0.80 \\
\hline \multicolumn{8}{|l|}{ In crude ash } \\
\hline $\mathrm{Ca}$ & 53.7 & 54.2 & 52.2 & 54.0 & 52.4 & 49.4 & 0.76 \\
\hline $\mathrm{P}$ & 36.6 & 36.1 & 35.6 & 36.1 & 36.1 & 34.0 & 0.53 \\
\hline $\mathrm{Mg}$ & 1.23 & 1.23 & 1.15 & 1.12 & 1.15 & 1.13 & 0.02 \\
\hline $\mathrm{Zn}, \mathrm{ppm}$ & 800.0 & 827.4 & 774.6 & 781.5 & 714.6 & 771.6 & 15.0 \\
\hline \multicolumn{8}{|c|}{ Measurement 2} \\
\hline Crude ash, $\%$ & 50.0 & 47.0 & 48.9 & 48.1 & 49.1 & 49.6 & 0.51 \\
\hline \multicolumn{8}{|l|}{ In crude ash } \\
\hline $\mathrm{Ca}$ & 52.7 & 56.9 & 54.1 & 55.0 & 52.9 & 51.9 & 0.80 \\
\hline $\mathrm{P}$ & 33.8 & 35.8 & 33.5 & 34.7 & 32.9 & 32.8 & 0.43 \\
\hline $\mathrm{Mg}$ & 1.00 & 1.02 & 1.02 & 1.01 & 1.01 & 0.94 & 0.02 \\
\hline $\mathrm{Zn}, \mathrm{ppm}$ & 802.8 & 870.9 & 850.8 & 819.9 & 831.9 & 853.6 & 11.49 \\
\hline \multicolumn{8}{|c|}{ Measurement 3} \\
\hline Crude ash, $\%$ & 47.1 & 46.6 & 50.5 & 48.7 & 48.1 & 50.7 & 0.62 \\
\hline \multicolumn{8}{|l|}{ In crude ash } \\
\hline $\mathrm{Ca}$ & 50.3 & 51.2 & 48.3 & 50.6 & 49.5 & 50.2 & 0.59 \\
\hline $\mathrm{P}$ & 34.5 & 34.8 & 31.1 & 33.9 & 34.7 & 34.2 & 0.52 \\
\hline $\mathrm{Mg}$ & $1.00^{\mathrm{AB}}$ & $1.04^{\mathrm{A}}$ & $0.86^{\mathrm{B}}$ & $0.99^{\mathrm{AB}}$ & $0.97^{\mathrm{AB}}$ & $1.01^{\mathrm{AB}}$ & 0.02 \\
\hline $\mathrm{Zn}, \mathrm{ppm}$ & $817.7^{\mathrm{AB}}$ & $829.8^{\mathrm{AB}}$ & $757.4^{\mathrm{A}}$ & $796.0^{\mathrm{AB}}$ & $834.2^{\mathrm{AB}}$ & $890.5^{\mathrm{B}}$ & 13.19 \\
\hline \multicolumn{8}{|c|}{ Means for three measurements } \\
\hline Crude ash, $\%$ & 47.0 & 46.4 & 48.7 & 47.9 & 48.4 & 50.2 & 0.39 \\
\hline $\mathrm{Ca}$ & 52.5 & 54.1 & 51.5 & 53.2 & 51.6 & 50.5 & 0.45 \\
\hline $\mathrm{P}$ & 35.3 & 35.6 & 33.4 & 34.9 & 34.6 & 33.7 & 0.30 \\
\hline $\mathrm{Mg}$ & 1.07 & 1.01 & 1.01 & 1.04 & 1.04 & 1.03 & 0.01 \\
\hline $\mathrm{Zn}, \mathrm{ppm}$ & 806.9 & 842.7 & 794.3 & 799.1 & 793.6 & 838.6 & 8.06 \\
\hline Means for measurements & \multicolumn{2}{|c|}{1} & \multicolumn{3}{|c|}{2} & \multicolumn{2}{|c|}{3} \\
\hline Crude ash, $\%$ & \multicolumn{2}{|c|}{$46.8^{\mathrm{A}}$} & \multicolumn{3}{|c|}{$48.8^{\mathrm{B}}$} & \multicolumn{2}{|c|}{$48.6^{\mathrm{B}}$} \\
\hline $\mathrm{Ca}$ & \multicolumn{2}{|c|}{$52.6^{\mathrm{a}}$} & \multicolumn{3}{|c|}{$53.9^{\mathrm{A}}$} & \multicolumn{2}{|c|}{$50.0^{\mathrm{Bb}}$} \\
\hline $\mathrm{P}$ & \multicolumn{2}{|c|}{$35.7^{\mathrm{a}}$} & \multicolumn{3}{|c|}{$33.9^{\mathrm{b}}$} & \multicolumn{2}{|c|}{$34.0^{\mathrm{b}}$} \\
\hline $\mathrm{Mg}$ & \multicolumn{2}{|c|}{$1.17^{\mathrm{A}}$} & \multicolumn{3}{|c|}{$1.00^{\mathrm{B}}$} & \multicolumn{2}{|c|}{$0.98^{\mathrm{B}}$} \\
\hline $\mathrm{Zn}, \mathrm{ppm}$ & \multicolumn{2}{|c|}{$778.3^{\mathrm{A}}$} & \multicolumn{3}{|c|}{$838.3^{\mathrm{B}}$} & \multicolumn{2}{|c|}{$820.9^{\mathrm{B}}$} \\
\hline
\end{tabular}

a,b,A,B means in a row with no common superscripts are significantly different: ${ }^{a, b} \mathrm{P}<0.05$; ${ }^{\mathrm{A}, \mathrm{B}} \mathrm{P}<0.01$ 
Phytase caused significant diversification of $\mathrm{Mg}$ and $\mathrm{Zn}$ content only in the third measurement period. The reasons for the lower concentration of $\mathrm{P}$ and the higher $\mathrm{Mg}$ concentration in the bones, which were observed in the second experimental period, are unclear and difficult to explain.

The coefficients of correlation that were calculated for bone strength and elasticity and $\mathrm{Ca}$ and $\mathrm{P}$ content in dry matter of bones varied insignificantly between 0.36 and 0.46 .

There were some significant differences between groups in $\mathrm{Ca}, \mathrm{P}, \mathrm{Mg}$ and $\mathrm{Zn}$ concentrations in crude ash, but they are inconclusive; however, the content of $\mathrm{Mg}$ in ash increased and the content of $\mathrm{Zn}$ declined with the age of the hens (Tables 8 and 9).

\section{DISCUSSION}

In the present study the low-phosphorus diet for layers was supplemented with two different phytase preparations, each of them was applied at two levels. The amount of available $\mathrm{P}$ was reduced to $1.89 \mathrm{~g}$ and then to $1.31 \mathrm{~g}$ per $\mathrm{kg}$ of feed as compared with $3.09 \mathrm{~g} / \mathrm{kg}$ in the control diet and it did not lead to a decrease of body weight or laying rate. These basic performance parameters were also not improved by phytase supplementation. The average laying rate for the total period of egg production was $92.7 \%$. The number and weight of eggs obtained were also not affected by the P level and did not depend on the kind or level of added phytase. Adult hens usually show a slight response to feed supplementation with biologically active substances (Jamroz et al., 1998). The results of the present study are in agreement with the reports of Htoo and Liebert (2001).

The daily feed intake in hens fed the low-P diets or diets supplemented with phytase B was similar; a significantly higher feed intake was recorded only in the group fed the diet with $450 \mathrm{U} / \mathrm{kg}$ phytase A. However, feed conversion was significantly better in the group fed the diet with $450 \mathrm{U} / \mathrm{kg}$ phytase $\mathrm{B}$. The possibility of improvement of feed conversion by phytase addition has been described by Hadorn and Wiedmer (1998) and Oloffs et al. (2000). One of the possible explanations of the low phytase efficacy in hens in the present experiment may be the high amounts of wheat and barley in the diet; these grains are characterized by relatively high native phytase activity (Liebert et al., 2001).

The phosphorus level or addition of phytase did not affect the percentage of cracked eggs. The highest shell thickness was found in eggs from the positive control (VI) and groups II and III in the first and third experimental period. Only in individual cases was a slight enhancement in this parameter registered, particularly when phytase B at a level of $450 \mathrm{U}$ or phytase A at level of 300 or $450 \mathrm{U} / \mathrm{kg}$ were applied. 
Phytases, enzymes degrading phytate in feeds, release mineral elements and improve $\mathrm{P}$ but also $\mathrm{Ca}$ conversion. This makes better egg shell mineralization possible and consequently improves the quality of eggs (Sauveur, 1991; Um and Paik, 1999). The averages, calculated for three measurements, show that a higher egg shell thickness was found in the positive control group and in groups fed low P diets supplemented with $450 \mathrm{U}$ phytase B or with $450 \mathrm{U}$ phytase A. Generally, the strength of egg shells was lower in comparison with results reported by Jamroz et al. (1998), which might be related to the genotype of the hens.

In contrast to the indistinct effects on egg shell quality, the tibia and femur strength was improved by phytase addition. The differences between groups were statistically significant. The average values for bone strength varied from 3.97 to $5.59 \mathrm{~N} / \mathrm{mm}^{2}(60-99 \mathrm{~N})$ for the tibia and from 3.54 to $5.62 \mathrm{~N} / \mathrm{mm}^{2}(58-97 \mathrm{~N})$ for the femur. However, Fleming et al. (1994) and Gordon and Short (2001) reported values ranging from 115 to $249 \mathrm{~N}$. Phosphorus utilization depends on nutrition, housing systems and strains of hens or broilers (Simons et al., 1990). The bone parameters can also be differentiated by the kind of determined indices (Quian et al., 1996; Zhang and Coon, 1997). The strength indices relating to the tibia and femur, expressed in $\mathrm{N} / \mathrm{mm}^{2}$, were lower in hens from group I and the best in group $\mathrm{VI}$, groups supplemented with $450 \mathrm{U} / \mathrm{kg}$ phytase $\mathrm{A}$ and $300 \mathrm{U} / \mathrm{kg}$ phytase $\mathrm{B}$, respectively. The elasticity of bones, low in group I, was clearly higher in the positive control, group VI, and in groups supplemented with phytase.

In contrast to the assessed egg parameters, only a small decrease in shell quality was observed (in the second period) as an effect of the level of available P/kg of the diet being reduced to 1.89 and then to $1.31 \mathrm{~g}$ from $3.09 \mathrm{~g}$. In the tibia and femur in the control group only a slight reduction in the elasticity coefficient in the third measurement period was found. It is clear that in older hens the bone parameters were better than in younger ones at the beginning of egg production. The same pattern was observed in the case of low P (I) and enzyme-supplemented diets. There was no tendency towards higher accumulation of $\mathrm{Ca}$ in bones. In contrast to $\mathrm{Ca}$, the phosphorus content of bones of hens fed low-P diets with phytases was higher in the first and third measurement periods. In the present study the variability of $\mathrm{Mg}$ and $\mathrm{Zn}$ content in bones caused some difficulties in interpretation of the obtained results, however, in the opinion of Yi et al. (1996a) the concentration of $\mathrm{Zn}$ in bones can be improved by phytase supplementation.

Comparison of some parameters of bone quality and chemical composition (Table 10) points to significant differences between the tibia and femur. The femur is significantly stronger and more elastic than the tibia. The content of $\mathrm{Ca}, \mathrm{P}, \mathrm{Mg}$ and $\mathrm{Zn}$ in the femur is higher, too. This indicates that the tibia is a weaker bone. It is much more exposed to mechanical injury, especially since it is positioned at an angle to the base. 
TABLE 10

Comparison of some parameters of bone quality, calculated per $100 \mathrm{~g}$ metabolic body weight $\left(\mathrm{BW}^{0.67}\right)$ and content of minerals in crude ash of bones

\begin{tabular}{lccc}
\hline Item & Femur & Tibia & SEM \\
\hline Cortex thickness, mm & 0.48 & 0.48 & 0.006 \\
Surface, $\mathrm{mm}^{2}$ & $10.71^{\mathrm{A}}$ & $10.08^{\mathrm{B}}$ & 0.158 \\
Strength, $\mathrm{N}$ & $80.46^{\mathrm{A}}$ & $73.45^{\mathrm{B}}$ & 1.310 \\
Strength to surface ratio, $\mathrm{N} / \mathrm{mm}^{2}$ & 4.73 & 4.59 & 0.068 \\
Elasticity alpha coefficient, $\mathrm{kg} / \mathrm{mm}^{2}$ & $3.54^{\mathrm{A}}$ & $3.99^{\mathrm{B}}$ & 0.076 \\
& & & \\
Content of minerals in crude ash, $\%$ & 52.2 & 51.2 & 0.372 \\
$\quad \mathrm{Ca}$ & 34.56 & 34.11 & 0.239 \\
$\mathrm{P}$ & $1.05^{\mathrm{A}}$ & $0.99^{\mathrm{B}}$ & 0.097 \\
$\mathrm{Mg}$ & $812.5^{\mathrm{A}}$ & $723.8^{\mathrm{B}}$ & 6.834 \\
$\mathrm{Zn}, \mathrm{ppm}$ & & & \\
\hline
\end{tabular}

In conclusion it can also be stated that the bone quality parameters of laying hens are more rational and useful indices for estimation of phosphorus availability and phytase efficiency than egg shell characteristics. A similar conclusion was drawn by Orban et al. (1999) and Um and Paik (1999).

\section{REFERENCES}

AOAC, 1990. Official Methods of Analysis, Association of Official Analytical Chemits. $15^{\text {th }}$ Edition. Arlington, VA

Brandt S., 1997. Statistical and Computational Methods in Data Analysis. $3^{\text {rd }}$ Edition. Springer Verlag, New York

De Groote G., Huyghebaert G., 1997. The bio-availability of phosphorus from feed phosphates for broilers as influenced by bio-assay method, dietary Ca level and feed form. Anim. Feed Sci. Tech. 69, 329-340

European Table of Energy Values for Poultry Feedstuffs, 1989. WPSA. Beekbergen (The Netherlands)

Fleming R.H., Whitehead C.C., Alvey D., Gregory N.G., Wilkins L.J., 1994. Bone structure and breaking strength in laying hens housed in different husbandry systems. Brit. Poultry Sci. 35, 651-662

Gordon S.H., Short F.J., 2001. The effect of phytase supplementation and limestone granules on tibia breaking strength of laying hens in cage systems. In: Proceedings of $13^{\text {th }}$ European Symposium on Poultry Nutrition, Blankenberge (Belgium), pp. 242-243

Hadorn R., Wiedmer H., 1998. Effekte einer Phytasezulage zu einem Phosphorreduzierten Legehennenfutter. Arch.Geflügelk. 62 (3), 97-103

Htoo J.K., Liebert F., 2001. Efficiency of phytate in layer diets without phosphorus supplementation. In: Proceedings 8. Symposium: Vitamine und Zusatzstoffe in der Ernährung von Mensch und Tier, Jena (Germany), pp. 477-480 
Jamroz D., Skorupińska J., Orda J., Wiliczkiewicz A., 1998. Influence of antibiotica promotors on the egg production and quality as well as nitrogen and phosphorus utilization in laying hens. Arch.Geflügelk. 62 (2), 1-9

Kornegay E.T., Denbow D.M., Yi Z., Ravindran V., 1996. Response of broilers to graded levels of microbial phytase added to maize-soya bean-based diets containing three levels of non-phytate phosphorus. Brit. J. Nutr. 75, 839-852

Leske K.L., Coon C.N., 1999. A bioassay to determine the effect of phytase on phytate phosphorus hydrolysis and total phosphorus retention of feed ingredients as determined with broilers and laying hens. Poultry Sci. 78, 1151-1157

Liebert F., Sukria H.A., Htoo J.K., 2001. Zur Wirksamkeit von Phytaseeränzungen bei differenzierter nativer Phytaseaktivität. In: Proceedings 8. Tagung "Schweine- und Geflügelernährung, Jena (Germany), pp. 261-266

Oloffs K., Cossa J., Jeroch H., 2000. Phosphorus utilisation from different vegetable feedstuffs by laying hens. Arch. Geflügelk. 64 (1), 24-28

Nutrient Requirements of Poultry. Nutritive Value of Feedstuffs (in Polish), 1996. 3rd Edition. The Kielanowski Institute of Animal Physiology and Nutrition (Editor). Jabłonna (Poland)

Orban J.I., Adeola O., Stroshine R., 1999. Microbial phytase in finisher diets of White Pekin ducks. Effect on growth performance, plasma phosphorus concentration and leg bone characteristics. Poultry Sci. 78, 366-377

Ravindran V., Cabahug S., Ravindran G., Bryden W.L., 1999. Influence of microbial phytase on apparent ileal aminoacid digestibility of feedstuffs for broilers. Poultry Sci. 78, 699-706

Sauveur B., 1991. Phosphorus metabolism in laying hens: effects of intermittent calcium and phosphorus supplies. In: Proceedings of $8^{\text {th }}$ European Symposium Poultry Nutrition, VeneziaMestre (Italy), pp. 213-228

Saynaeve M., Janssen G., Hesta M., Van Nevel C., De Wilde R.O., 2000. Effects of dietary Ca/P ratio, $\mathrm{P}$ level and microbial phytase supplementation on nutrient digestibilities in growing pigs: breakdown of phytic acid, partition of $\mathrm{P}$ and phytase activity along the intestinal tract. J. Anim. Physiol. Anim. Nutr. 83, 193-204

Sebastian S., Touchburn S.P., Chavez E.R., Laque P.C., 1996. The effects of supplemental microbial phytase on the performance and utilization of dietary calcium, phosphorus, copper and zinc in broiler chickens fed corn-soybean diets. Poultry Sci.75, 729-736

Simons P.C.M., Versteegh H.A.J., Jongbloed A.W., Kemme P.A., Slump. P., Bos K.D., Wiolters M.G.E., 1990. Improvement of phosphorus availability by microbial phytase in broilers and pigs. Brit. J. Nutr. 64, 525-540

Statgraphics Plus ver. 7 for DOS, 1993. Statistical Graphics System

Szkudelski T., 1997. Phytic acid - its influence on organism. J. Anim. Feed Sci. 6, 427-438

Quian H., Kornegay E.T., Veit H.OP., 1996. Effects of supplemental phytase and phosphorus on histological, mechanical and chemical traits of tibia and performance of turkey fed on soya bean meal-based semi-purified diets high in phytate phosphorus. Brit. J. Nutr. 76, 263-272

Um J.S., Paik J.K., 1999. Effects of microbial phytase supplementation on egg production, egg shell quality and mineral retention of laying hens fed different levels of phosphorus. Poultry Sci. 78, 75-79

Van der Klis J.D., 1991. Factors affecting the absorption of minerals from the gastro-intestinal tract of broiler. In: Proceedings of $8^{\text {th }}$ European Symposium Poultry Nutrition, Venezia-Mestre (Italy), pp. 242-255

Yi Z., Kornegay E.T., Denbow D.M., 1996a. Supplemental microbial phytase improves zinc utilization in broilers. Poultry Sci. 75, 540-546 
Y i Z., Kornegay E.T., Denbow D.M., 1996b. Effect of microbial phytase on nitrogen and amino acids digestibility and nitrogen retention of turkey poults fed corn-soybean meal diets. Poultry Sci. 75, 979-990

Zhang B., Coon C.N., 1997. The relationship of various tibia bone measurements in hens. Poultry Sci. 76, 1698-1701

Żyła K., Koreleski J., Kujawski M., 1989. Dephosphorylation of phytate compounds by means of acid phosphatase from Aspergillus niger. J. Sci. Food Agr. 49, 315-324

Żyła K., Ledoux D.R., Kujawski M., Veum T.L., 1996. The efficacy of an enzymatic coctail and a fungal mycelium in dephosphorylating corn-soybean meal-based feeds fed to growing turkeys. Poultry Sci. 75, 381-387

\section{STRESZCZENIE}

Reakcja kur niosek na obniżony poziom fosforu oraz dodatek różnych preparatów fitazy do mieszanek

Celem badań było określenie wpływu dodatku dwóch preparatów fitazy do mieszanek o obniżonej zawartości dostępnego fosforu na produkcję jaj, pobranie paszy, jakość skorupy jaj, wytrzymałość i elastyczność kości i zawartość w nich Ca, P, Mg i Zn. Kurki Lohmann Brown w wieku 16 tygodni przydzielono do 6 grup, z których każda obejmowała 12 klatek po 4 ptaki. Przed rozpoczęciem nieśności ptaki żywiono mieszanką standardową zawierającą $150 \mathrm{~g}$ białka ogólnego, 11,3 MJ EM i 4,0 g P przyswajalnego w $\mathrm{kg}$. Mieszanki doświadczalne sporządzono z pasz roślinnych, $\mathrm{w}$ tym około $70 \%$ stanowiły pszenica i jęczmień. Mieszanki zawierały $165 \mathrm{~g}$ białka ogólnego, $11,2 \mathrm{MJ}$ EM $/ \mathrm{kg}$. W dietach I-V ilość P dostępnego wynosiła 1,89 g do 14 tygodnia nieśności, a następnie $1,31 \mathrm{~g} / \mathrm{kg}$, dieta kontrolna VI zawierała 3,09 g P dostępnego/kg. Dwa rodzaje fitazy (A i B) dodano w ilości 300 lub 450 jedn./kg mieszanki do diet II-V. Dodatek fitazy do mieszanek niskofosforowych nie wpłyną na wskaźniki produkcji jaj. Tylko zastosowanie 450 jedn./kg fitazy B poprawiło wykorzystanie paszy. W odróżnieniu od parametrów jakości skorupy jaj, dodatek fitazy istotnie poprawił wskaźniki wytrzymałości i elastyczności kości podudzia i uda, które były wyższe lub podobne jak w grupie kontrolnej (VI). 